\begin{tabular}{|c|c|c|c|c|c|}
\hline & $\begin{array}{l}\text { Baseline } \\
\text { AF }\end{array}$ & $\begin{array}{l}\text { Baseline } \\
\mathrm{HF}\end{array}$ & $\begin{array}{l}\text { BaselineAF } \\
+\mathrm{HF}\end{array}$ & $\begin{array}{l}\text { Baseline AF, } \\
\text { developed HF }\end{array}$ & $\begin{array}{l}\text { Baseline } \mathrm{HF}, \\
\text { developed AF }\end{array}$ \\
\hline$n$ & 29164 & 19474 & 5728 & 1647 & 824 \\
\hline $\mathrm{Age} \pm \mathrm{SD}$ & $74 \pm 13$ & $73 \pm 14$ & $77 \pm 12$ & $77 \pm 11$ & $77 \pm 11$ \\
\hline Male\% & 52.4 & 51.0 & 49.8 & 48.0 & 49.3 \\
\hline Caucasian\% & 87.6 & 82.9 & 86.8 & 92.2 & 90.2 \\
\hline South Asian\% & 1.7 & 4.0 & 1.8 & 1.6 & 2.4 \\
\hline $\begin{array}{l}\text { Afro-Caribbean } \\
\%\end{array}$ & 0.8 & 1.6 & 1.2 & 0.4 & 0.6 \\
\hline Other\% & 9.9 & 11.8 & 10.2 & 5.8 & 6.8 \\
\hline $\begin{array}{l}\text { Crude } \\
\text { Mortality (per } \\
1000 \text { ) }\end{array}$ & 485 & 636 & 672 & 715 & 778 \\
\hline $\begin{array}{l}\text { Mean Survival } \\
\text { (Days) }\end{array}$ & 722 & 631 & 692 & 880 & 922 \\
\hline
\end{tabular}

$\mathrm{AF}$, atrial fibrillation; $\mathrm{HF}$, heart failure; $\mathrm{SD}$, standard deviation

developed HF during follow-up, and of those with $\mathrm{HF}$ at baseline, 824 (4.2\%) developed AF during follow-up. Demographics and crude mortality rates are shown; see Table. Patients with combined $\mathrm{AF}$ and $\mathrm{HF}$ at baseline had increased mortality than patients with $\mathrm{AF}$ or $\mathrm{HF}$ alone. Patients with $\mathrm{AF}$ at baseline that developed HF, and patients with HF at baseline that developed AF, experienced a greater mortality compared to those with combined $\mathrm{HF}$ and $\mathrm{AF}$ at baseline; see Figure.

Conclusion Concomitant AF and HF is associated with substantial mortality and risk of death, irrespective of which disease develops first. In light of limited current treatment for these patients, future therapies to specifically target the combined $\mathrm{HF}$ and $\mathrm{AF}$ group are required.

\section{AUDIT OF MANAGEMENT OF PATIENTS WITH HEART FAILURE FOLLOWING NON-STEMI AND USE OF ALDOSTERONE ANTAGONISTS}

${ }^{1}$ Olivia Buckledee*, ${ }^{2}$ Yasmin Ismail, ${ }^{2}$ Angus Nightingale. ${ }^{1}$ University of Bristol; ${ }^{2}$ Bristol Heart Institute; *Presenting Author

\subsection{6/heartjnl-2016-309890.22}

Background The presence of heart failure following a non-ST elevation myocardial infarction (non-STEMI) is associated with a worse prognosis. Current UK and European Guidelines recognise the importance of identifying these higher risk patients and offering them disease modifying therapies including drugs and devices.

We wanted to find out of these guidelines were being followed in a busy Heart Attack Centre in the South West of England; in particular, whether a mineralocorticoid receptor antagonist (MRA) was being prescribed to those with impaired LV function and either diabetes or signs heart failure.

Methods We performed a retrospective audit of consecutive patients identified as having non-STEMI on the MINAP database form April 2014 to March 2015.

We assessed:

1. How many received an echocardiogram during their index admission;
2. Compliance with NICE recommended post MI medications on discharge;

3. Implications for device implantation rates according to NICE and ESC guidelines.

Results 176 patients (mean age 70 years, 64\% male) were recorded on the MINAP database with non-STEMI in the study period. $71 \%(125 / 176)$ had an echocardiogram during the index admission. 42\% (53/125) had good left ventricular (LV) function and $30 \%(38 / 125)$ had moderately or severely impaired LV function ( $\mathrm{EF}<40 \%)$.

Of those with impaired LV function post non-STEMI, 82\% (31/38) either had diabetes or signs of pulmonary oedema; 6 of these had a documented contra-indication to a MRA. 56\% $(14 / 25)$ were prescribed a MRA in accordance with the NICE guidelines compared to $>95 \%$ compliance with guidelines recommending ACEi/BB/statin/antiplatelet agents. Mean length of stay for those with impaired LV function was 7.9 days. $71 \%$ (27/38) of the impaired LV function patients were followed up by our cardiology team with 11 referred back to their local hospital for follow up. $41 \%(11 / 27)$ had a repeat echo after at least one month to reassess LV function and assess requirement for implantable devices.

Conclusions Nearly a third of patients had significantly impaired LV function following non-STEMI. Whilst most postMI drugs were prescribed $>95 \%$ in compliance with the NICE guidelines, MRAs (spironolactone and eplerenone) were only prescribed in around half of suitable cases. The reasons for this could include poor documentation of contraindication to MRA or lack of awareness of the guidelines.

Follow-up reassessment of LV function after at least a month to detect functional recovery or indication for device therapy was also poor. Opportunities to prevent sudden death or worsening heart failure by implanting ICD or CRT devices could therefore have been missed.

Management of non-STEMI patients complicated by heart failure might benefit from a closer link between the acute coronary syndrome and the heart failure teams to improve outcomes and reduce length of stay for patients.

\section{SURVEILLANCE AND INCIDENCE OF CHEMOTHERAPY- INDUCED CARDIOTOXICITY IN BREAST CANCER: A LONG TERM OBSERVATIONAL STUDY}

Bayan Soujeri*, Jagdeep Singh, Samuel Chew, Sean Hawkey, Michelle Ferguson, Chim C Lang. University of Dundee; *Presenting Author

\subsection{6/heartjnl-2016-309890.23}

Introduction Recent advances in chemotherapy have transformed breast cancer from a fatal disease to a survivable condition. However, many patients who survive their cancer, succumb to the unintended adverse effects of therapy. Chemotherapy-induced cardiotoxicity is now among the most feared adverse effects and has been reported in up to $50 \%$ of patients up to twenty years later, the majority of which occurring after completion of therapy. Consequently, current breast cancer treatment guidelines recommend assessment of LV function before, during and after chemotherapy.

Methods We conducted a retrospective cohort study of 1263 breast cancer patients who received either anthracyclines alone or combined with the monoclonal antibody trastuzumab at Ninewells Hospital, Dundee between January 2003 and December 2014. Imaging modalities such as MUGA scans 
were extracted from the CHEMOCARE database which allowed us to identify whether cardiac assessments were carried out at baseline and during treatment as per recommendations. LVEF at baseline and during routine follow-up was also calculated to determine if patients had developed left ventricular systolic dysfunction (LVSD) during chemotherapy.

Results Of 1262 patients, 1260 patients (99.8\%) were female, with an mean age of 59.3 years. $82.2 \%$ of the patients had received anthracyclines alone and $17.8 \%$ received a combination of an anthracycline and trastuzumab. 900 patients (71.3\%) received adjuvant radiotherapy, 311 (24.6\%) received chemotherapy only and $51(4.0 \%)$ had an unknown type of therapy. $1101(87.2 \%)$ of patients were on epirubicin with an average cumulative dose of $570.7 \mathrm{mg} / \mathrm{m}^{2}$, making it the most used anthracyline as it is less cardiotoxic compared to doxorubicin. $683(54.0 \%)$ underwent screening for baseline cardiac function using MUGA scans. Of these, only 290 (42.5\%) had follow up scans during the course of their treatment as mandated by guidelines. Among patients who had adequate follow up, 32 (11\%) developed LVSD during chemotherapy, corresponding to a rate of $6.7 \%(n=5)$ among those on anthracyclines alone and $12.5 \%(\mathrm{n}=27)$ among patients on combination therapy.

Conclusion Despite recommendations to monitor cardiac function, our data shows in all chemotherapy patients, only half of them were monitored in our cohort. Although LVSD is usually a late sequel of cancer treatment, we have shown its occurrence even when chemotherapy is being delivered, thus highlighting the importance of surveillance to ensure early detection and management. This may reflect physicians' opinion, contrary to guideline recommendations, that single chemotherapy is associated with less cardiotoxicity.

\section{$24 \quad$ AN EPIDEMIC OF HFPEF?}

${ }^{1}$ Alexandra Thompson*, ${ }^{2}$ Jenifer Crilley, ${ }^{3}$ Douglas Wilson, ${ }^{3}$ APS Hungin, ${ }^{2}$ Ahmet Fuat, ${ }^{2}$ Jerry Murphy. ${ }^{1}$ HENE Cardiology ST6; ${ }^{2}$ County Durham and Darlington NHS Foundation Trust; ${ }^{3}$ Durham University; *Presenting Author

\subsection{6/heartjnl-2016-309890.24}

Introduction There is debate about the diagnostic profile of the heart failure population. Diagnostic criteria for heart failure with reduced ejection fraction (HFREF) are variable and many believe that heart failure with preserved ejection fraction (HFPEF) is over-diagnosed. There is also differing opinion about whether these conditions are distinct entities. The aim of this study was to differentiate a heart failure population with contemporary diagnostic parameters.

Methods A diagnostic pathway using current best evidence for the diagnosis of HFREF and HFPEF was created. This was applied prospectively to all 319 new patients attending our heart failure clinic between May 2013 and July 2014.

Results 245 of the 319 new patients were deemed to have heart failure clinically. $73 \%$ met our diagnostic criteria for HFREF (LVEF <55\%), and only 14\% met the diagnostic criteria for HFPEF. Changing the LVEF threshold to $<40 \%$ produced a ratio of HFREF to HFPEF of 1.3:1 which is more in keeping with current perceptions about an epidemic of HFPEF, but reflects the fact that many of those diagnosed

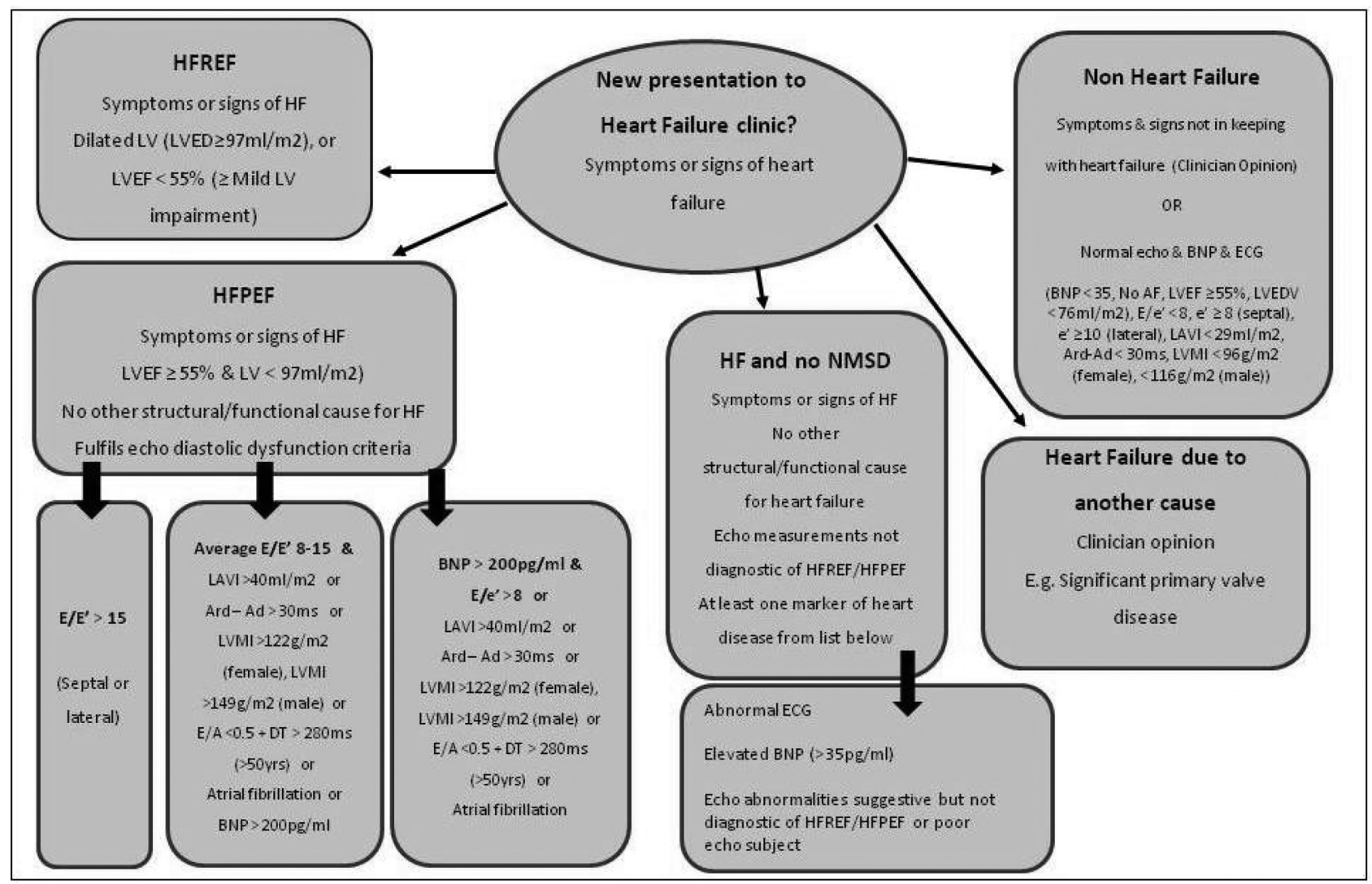

Abstract 24 Figure 1 HFREF, Heart failure with reduced ejection fraction; HFPEF, Heart failure with preserved ejection fraction; HFNMSD, Heart failure with no major structural disease; LVEF, Left ventricular ejection fraction 